\title{
Construction de l'événement médiatique à l'exemple de la crise des migrants en Europe. Vers une dimension argumentative
}

\section{The structure of a media event based on the example of the European migrant crisis. Argumentative model assessment}

\author{
Jolanta Dyoniziak \\ Université Adam Mickiewicz \\ joladyo@amu.edu.pl
}

\begin{abstract}
In this publication we would like to consider the issue of discursive strategy responsible for mediatisation of the migrant crisis currently taking place in Europe, partly as a result of military actions in the Middle East. The first objective is to describe media representation of the European migrant crisis published in the French and Polish press over the last five years. The author especially emphasizes one discursive moment, year 2015, when a significant increase in the number of migrants arriving in Europe occurred. The next task will be to assess the argumentative potential that results from a particular staging of the discussed event.
\end{abstract}

Keywords: media event, press discourse, mediatisation of migrant crisis, argumentative model.

\section{OBJET ET MÉTHODOLOGIE DE RECHERCHE}

La présente publication vise à l'analyse du discours d'information médiatique, à savoir du phénomène de la construction de l'événement médiatique qui y est saillant par une mise en scène, cette dernière constituant une stratégie discursive complexe dans laquelle les éléments stylistiques jouent un rôle important.

Les recherches en $\mathrm{AD}$ centrées sur le concept d'événement médiatique (Calabrese, 2013 ; Sini, 2015 ; Veniard, 2013) nous ont incitée à réfléchir sur la question des 
stratégies discursives responsables de la médiatisation de la crise des migrants que connaît l'Europe à l'heure actuelle et qui est imputable aux guerres civiles frappant le Moyen-Orient. Le premier but est de décrire la représentation médiatique de la crise des migrants en Europe, telle qu'elle apparaît dans la presse d'information française et polonaise durant les cinq dernières années, mais en mettant l'accent sur un moment discursif particulier, celui de l'année 2015 où l'on observa une importante augmentation du nombre des migrants venus en Europe.

L'information médiatique n'échappe pas aux enjeux argumentatifs, surtout quand il est question d'un événement qui marque tant la vie sociale. Du moment que les instances médiatiques s'engagent dans un débat social, ils proposent aux allocutaires des modèles de perception. Admis par certains, réfutés par d'autres, ils restent pourtant dans l'espace communautaire et peuvent s'introduire dans la doxa, d'autant plus qu'ils véhiculent des valeurs partagées. Ainsi, la question suivante sera de saisir et d'évaluer la dimension argumentative (celle-ci dans le cadre de la pragmatique intégrée : Amossy, 2006; Anscombre, 1995) de la mise en scène de l'événement indiqué.

Le corpus qu'on a établi afin de réaliser notre objectif est constitué d'éléments verbo-icôniques, conformément à la thèse posée par Lugrin (2001), selon laquelle l'information médiatisée apparaît sous forme de mosaïque sémiotique. Ils proviennent de la presse d'information accessible via Internet ainsi que des portails d'information français et polonais. Le choix porta sur des instances médiatiques dont la portée informationnelle est élevée et dont les profils idéologiques sont variés.

Les données ont été recueillies selon la méthode proposée par Calabrese (2009, 2013) et Veniard (2013). La sélection en fonction d'un mot-pivot (crise des migrants en Europe) nous a permis d'accéder à un nombre important de couvertures médiatiques. On y assiste à l'apparition de nombreuses « configurations événementielles » (Sini, 2015, para. 21) qui contribuent à un vrai programme de sens attribué à l'événement en question. La presse française et polonaise suive un schéma discursif divergent en plusieurs points, ce qu'illustre le matériel linguistique recueilli dans le corpus ainsi que les résultats de l'analyse.

\section{CRISE MIGRATOIRE EN EUROPE. CONTEXTE SOCIO-POLITIQUE}

La crise migratoire en Europe commença dans les années 2010 avec l'augmentation du nombre de migrants venant d'Afrique, du Moyen-Orient et d'Asie du Sud via la mer Méditerranée et les Balkans. À partir de l'année 2015, on assiste à une véritable crise car des réfugiés syriens venant de Turquie et du Liban se joignirent à ce mouvement, ce qui amplifia le phénomène et provoqua une crise à l'échelle européenne. 
Selon Frontex, durant la première moitié de l'année 2015, 350000 personnes sont entrées illégalement dans l'espace Schengen. Le HCR ${ }^{1}$ note une grave augmentation du nombre des demandes d'asile (437 384 en 2015). Le total des personnes venues en Europe par voies maritime et terrestre en 2015 dépasse le million ${ }^{2}$. En ce qui concerne le contexte politique du problème évoqué, il est fondé de souligner que ce mouvement migratoire massif plonge l'Europe dans une crise politique. De nombreuses divisions et des tensions diplomatiques importantes apparaissent entre les pays d'Europe, ainsi il n'y a pas d'accord sur l'attitude commune à adopter. Des pays leaders au sein de l'Union Européenne prônent pour la répartition du nombre de migrants entre les pays membres, et cela au nom du principe de solidarité, tandis que les pays d'Europe de l'Est s'y opposent.

Les tentatives de migration vers l'Europe ont augmenté à la suite des guerres civiles (notamment la guerre civile syrienne), des problèmes politiques en Turquie, de l'aggravation du conflit en Libye. Les conflits armés, les persécutions ou, tout simplement, la pauvreté ont contribué à l'augmentation des départs depuis de nombreux pays d'Afrique et d'Asie. Parmi ceux qui sont les plus représentés, le HCR note : la Syrie, l'Afghanistan et l'Irak, pourtant la liste en englobe encore d'autres pays $^{3}$.

\section{MÉDIATISATION DE LA CRISE MIGRATOIRE DANS LA PRESSE}

\subsection{TRAITEMENT MÉDIATIQUE DE L'ÉVÉNEMENT. ÉVÉNEMENT VERSUS FAIT}

Les sociologues soulignent la dualité de la connaissance de l'homme contemporain due à deux expériences différentes du monde : directe, quand celui-ci est témoin de l'événement et indirecte, lorsque cette connaissance se réalise par le biais des médias (Kluszczyński, 2008; Thomson, 1995). Cela a son reflet dans la théorie du discours médiatique. On y parle de l'événement médiatique à la différence d'un fait réel (Sini, 2015). L'événement constitue le concept central de la mise en discours, pourtant il est nécessairement ancré dans la dynamique phénoménologique en prolongeant la dualité entre factualité et discursif. Ainsi, l'événement résulte d'une construction sociale et discursive que les médias entreprennent au sein des sociétés et auxquelles ils servent de support tout au long du processus de médiatisation de l'information (ibidem, para. 6). L'événement est un concept secondaire par rapport à celui d'un fait et simultanément opérationnel vu son contexte d'application, puisqu'il sert à la distinction méthodologique entre l'espace sémantique créé dans

\footnotetext{
${ }^{1}$ Le Haut Commissariat des Nations unies pour les réfugiés.

${ }^{2} \mathrm{http}: / /$ www.unher.org/

${ }^{3} \mathrm{http}: / /$ www.unhcr.org/
} 
les médias et l'espace événementiel réel dont les dimensions ne sont pas nécessairement de nature publique. L'important est de souligner que parmi toute une série de faits, seulement certains peuvent devenir un événement médiatique. Sini (2015) définit le fait en tant qu'entité physique brute initiale, une donnée objective, nonréfléchie puisque antérieure à toute interprétation. Par contre, l'événement apparait comme un fait choisi parmi d'autres, donc c'est celui qui a subi une perception publique, c'est-à-dire « une saisie perceptive et interprétative » (Charaudeau, 2005). Dans le flux des changements continus dus à la vie quotidienne, souvent routiniers, certains font irruption dans l'espace public (Sini, 2015, para. 10) et sont soumis à un jugement. Une fois mis dans un discours, c'est ainsi qu'ils deviennent un objet de pensée (ibidem, para. 11). Pour en donner une définition complète, il faut insister sur les points suivants :

- l'événement surgit dans l'espace public,

- il constitue un fait choisi parmi d'autres,

- il est un objet de pensée selon « les pratiques sociales, dans un contexte historique, social, culturel et symbolique » (Veniard, 2013, p. 24).

L'événement médiatique est défini comme un événement qui se déroule dans l'espace public, et qui fut sélectionné par une instance ou un agent dont l'activité professionnelle est liée aux médias et soumis à une sémiotisation dans un contexte social particulier, par laquelle il accède à un certain sens social (Calabrese, 2013). L'événement médiatique compris ainsi constitue notre concept opérationnel qui sert de support méthodologique à l'analyse effectuée.

\subsection{TRAITEMENT MÉDIATIQUE DE LA CRISE DES MIGRANTS EN EUROPE : PRINCIPAUX CONSTATS ET ANALYSE DU CORPUS}

Plusieurs discours prolifèrent sur la crise des migrants en Europe, ainsi le sujet est-il vivement en débat. Son identification en tant qu'événement médiatique marquant la réalité sociale de l'Europe se fait par la mise en catégorie majeure, celle de la crise, ce qui, selon Calabrese (2011, para. 20), constitue la première étape de la démarche dénominative inaugurant la sémiotisation du référent. La construction du sens social est premièrement due à la catégorisation, pourtant le processus est beaucoup plus complexe. Pour que la réception de l'événement se matérialise au sein d'une collectivité, autrement dit, pour que l'événement acquière le statut partagé (Veniard, 2007, p. 17), les instances médiatiques ont recours aux constructions logico-argumentatives responsables de la mise en scène ainsi que de l'interprétation de l'événement. Cette construction, une fois mise en pages, ouvre la voie à tout un programme de sens qui se réalise dans les discours et qui exploite la praxis des vocables utilisés (Siblot, 2001, para. 10) ainsi que d'autres supports discursifs non-verbaux, entre autres, la photographie. 
La médiatisation de la crise des migrants en Europe relève d'abord des enjeux éthiques, son interprétation est fortement ancrée dans l'idéologie démocratique et fait référence aux valeurs de liberté, d'égalité ainsi que du respect pour la paix. Ces valeurs sont admises comme principes constitutifs des sociétés européennes modernes. Le problème évoque la question de l'assimilation des étrangers, celle-ci étant souvent mise en doute vu les expériences de l'Europe occidentale face à l'identité des migrants fortement marquée par un système culturel exogène. Pourtant, le problème n'échappe plus aux enjeux économiques et politiques puisque l'arrivée d'un nombre excessif de migrants a des répercussions profondes sur l'économie des pays d'accueil ainsi que sur leur organisation politique. Pour faire adhérer les allocutaires aux représentations faites, les instances médiatiques situent ces dernières dans un espace d'opinions et de croyances collectives. Ainsi, les points de vue véhiculés se consolident aisément et gagnent l'ethos grâce auquel ils peuvent s'introduire dans le savoir commun (Amossy, 2006, pp. 99-100). L'analyse du matériel recueilli nous pousse à faire une remarque selon laquelle le discours sur la crise des migrants en Europe est porteur de schémas perceptifs établis par le biais d'analogies à des faits connus en tant que véritables fléaux : inondation et guerre. L'analogie se construit sur la similarité des caractéristiques de la crise des migrants et des indices conceptuels propre à ces deux événements. Les agents qui entrent en scène, l'espace concerné et les dimensions sont similaires au point que l'événement d'arrivée est enregistré en tant qu'occurrence du concept événementiel. Le mécanisme est simple, il consiste en une reprise d'idées; selon Amossy " Le nouveau est mis en perspective et éclairé par le familier et l'ancien » (2006, p. 146). Le recours aux formes topiques apporte à la représentation faite un avantage de nature axiologique, le schéma perceptif implique l'occurrence des valeurs qui proviennent d'une conscience collective. Les deux schémas actualisés au moment de la médiatisation de la crise des migrants impliquent des jugements de valeur négatifs liés à un danger pour l'être humain difficile à saisir et entraînant nécessairement des pertes. L'enchaînement argumentatif s'établit à l'aide d'une série de topos $(\mathrm{P}+\mathrm{Q})$ où $\mathrm{P}$ signifie l'objet et $\mathrm{Q}$ les traits qui y sont associés, dans le cas analysé, ce sont : détérioration, dégradation, malheur, mal, ravage, déchéance, chute, mort, etc. Ainsi, le discours acquière-t-il une potentialité idéologique, celle-ci est fortement ancrée dans la doxa des civilisations occidentales (Amossy \& Herschberg Pierrot, 1997). La stratégie discursive mise en place afin de créer la représentation des faits en question actualise généralement ces deux scénarios argumentatifs ou topoï (Anscombre, 1995) et donne à l'événement un éclairage particulier. On ne peut pas dissocier ce dernier des effets de nature argumentative puisque, comme l'analyse le prouve, il y contribue manifestement. Il procure au discours d'information médiatique une vraie dimension argumentative et le situe à l'opposé du non-argumentatif (Amossy, 2006, pp. 32-34), contrairement aux engagements éthiques de la démarche informative. 


\subsubsection{TOPOÏ DE L'EAU \\ (ANALOGIE À L'INONDATION, FORCE NATURELLE QUI MENACE LA CIVILISATION OCCIDENTALE)}

La construction de la représentation sociale de la crise évoquée s'effectue à l'aide d'un cadre métaphorique à deux agents. Le premier, les migrants, est fortement actif à la différence du second, celui de l'Europe, qui est passif. Les deux agents sont visiblement collectifs. Le premier constitue une quantité indéterminée, donc une masse de gens cherchant l'asile à la suite des conflits ayant lieu sur leurs territoires. La situation est dénommée à l'aide des lexèmes suivants : vague, afflux, fala uchodźców, zalew (1-7). Les paradigmes qualificatifs dominants sont ici : nombre imprécis, indéfini ou sans limites d'individus anonymes. Les mots dénotant un écoulement de l'eau, un mouvement et un déplacement agité par le vent ou par toute autre impulsion, comme par exemple les mouvements tectoniques, impliquent un contenu connotatif bien particulier, celui d'un exode massif et spontané des populations, impossible à contrôler qui échappe à tout essai de saisissement et de limitation que l'homme peut entreprendre.

(1) Fala uchodźców zalewa Serbię (...) (fakt.pl, le 27 août 2015) [fr. La vague des réfugiés inonde la Serbie]

(2) Fala idzie na zachód (wyborcza.pl, le 4 septembre 2015) [fr. La vague se dirige vers l'ouest]

(3) Musimy rozmawiać z diabłem, czyli jak powstrzymać falę uchodźców (wyborcza.pl, le 23 octobre 2015) [fr. Nous devons nous entretenir avec le diable, ou comment arrêter la vague de réfugiés]

(4) Europe : la vague de migrants grossit encore, les Européens étalent leurs divisions (lexpress.fr, le 10 septembre 2015)

(5) Vague de migrants en Grèce : renforts policiers sur l'île de Kos (lapresse.ca, le 12 août 2015)

(6) L'Europe confrontée à un afflux inédit de migrants traversant la Méditerranée selon l'ONU (lemonde.fr, le 7 juillet 2015)

(7) À Lesbos, les migrants continuent d'affluer (lemonde.fr, le 9 janvier 2016)

Le second agent paru dans ce cadre est visiblement patient, il subit l'action entreprise par le premier, ce qui est explicité au niveau du discours à l'aide des tournures passives. Dans la majorité des cas, ce sont des constructions avec un complément d'agent explicite (8-11), contrairement aux smalls clauses. L'allégement de la structure passive par l'ellipse de l'auxiliaire être ne nuit pas à l'instauration entre deux arguments d'une relation actancielle d'agent (migrants) à patient (Europe). La position de complément d'agent est marquée au moment où elle acquiert une valeur rhématique, ainsi le groupe constituant le propos ( $8:$ zalana imigrantami $)$ s'impose sur le thème discursif $(8:$ Italia $)$. Le schéma est fréquemment repris, ce que prouvent les exemples suivants (9-11). Les constructions impliquent une lecture résultante 
du procès subi puisque les formes verbales de départ sont perfectives (pol. zalać, fr. déborder, submerger) :

(8) Italia zalana imigrantami (gosc.pl, le 8 juin 2015) [fr. L’Italie débordée par les migrants]

(9) L'Allemagne débordée par l'afflux de réfugiés (lepoint.fr, le 6 mars 2015)

(10) La Slovénie à son tour débordée par les migrants (lefigaro.fr, le 21 octobre 2015)

(11) La Suède submergée par l'afflux de migrants rétablit ses contrôles frontaliers (lepoint.fr, le 12 novembre 2015)

Le résultat du procès est présenté à l'aide des mots en emploi métaphorique : naufrage, zalew (fr. un afflux) (12-15), potop (fr. un déluge) (16), tsunami (fr. un tsunami) (17-19) qui connotent par hyperbole un accident grave, une perte importante, un énorme drame, celui-ci contribuant à l'annihilation de la matière vivante, ici de l'organisation socio-politique et culturelle de l'Europe. La dimension illocutoire qui accompagne le contenu propositionnel instaure l'idée de menace en vigueur et de destruction de sa stabilité politique et culturelle.

(12) L'Europe, le continent qui se noie dans la crise des réfugiés (lemonde.fr, le 21 mars 2016)

(13) Méditerranée : un naufrage européen (liberation.fr, le 19 avril 2015)

(14) Un naufrage européen (lefigaro.fr, le 19 avril 2015)

(15) Migrants en Méditerranée : un naufrage européen (tempsreel.nouvelobs.com, le 22 avril 2015)

(16) „Imigranci w Europie to swego rodzaju potop” (www.newsjs.com, le 8 septembre 2015) [fr. Les immigrés en Europe constituent une sorte de déluge]

(17) Ludzkie tsunami uderzyło na Włochy (gosc.pl, le 4 avril 2011) [fr. Un tsunami humain a touché l'Italie]

(18) Berlusconi o imigrantach z Afryki: Ludzkie tsunami (newsweek.pl, le 1 avril 2011) [fr. Berlusconi sur les immigrants d'Afrique : un tsunami humain]

(19) Immigration : l'Europe face au tsunami humain des réfugiés (www.jeuneafrique. com, le 7 septembre 2015)

L'apport iconique est de grande importance dans la médiatisation du problème de la migration vers l'Europe. Les photos ont une fonction de co-construction du discours ainsi que de sa légitimation. Certaines images sont de nature reportage, ainsi instaurent-elles le registre du savoir, tandis que d'autres, puisqu'elles gardent la nature symbolique, véhiculent le registre modalisé, celui d'opinion.

Quant à la première catégorie, on y voit souvent des boat-people ${ }^{4}$ avec, à leurs bords, des nuées de réfugiés espérant rejoindre l'Europe, et cela malgré l'insécurité.

\footnotetext{
${ }^{4}$ Les boat-people, terme apparu dans la presse francophone dans les années soixante-dix, il désigna les migrants : Vietnamiens, Cambodgiens et Laotiens qui fuyaient par voie de mer les régimes communistes s'installant à l'époque sur leurs territoires.
} 
Toute image explique à quel point les bateaux, souvent juste des canots pneumatiques, sont surchargés, et quel est le risque que les réfugiés prennent afin de commencer une meilleure vie dans laquelle la précarité, l'incertitude et l'insécurité seront manifestement diminuées. Le plan d'ensemble ainsi que le plan moyen y sont dominants. Le premier laisse voir le décor, ici la mer, le second porte plus l'attention sur les actants, principalement sur les groupes des migrants. Souvent les photos cadrent une action en cours (fakty.interia.pl, le 26 août 2015 ; wyborcza.pl, le 2 juin 2015), une opération de sauvetage en mer Méditerranée (lemonde.fr, le 21 mars 2016; wyborcza.pl, le 28 mars 2016), de violents affrontements avec la police à la frontière (lapresse.ca, le 12 août 2015).

La photo symbolique cadre de préférence un événement dont la portée significative ne peut pas être niée. Le jeu de couleur y devient plus raffiné ainsi que le choix de l'image dans le cadre : une masse de personnes anonymes dont on voit seulement des figures à l'ombre puisque la photo est prise à contre-jour (lefigaro.fr, le 21 octobre 2015), un tas de gilets de sauvetage laissés sur la plage, dont la couleur orange, criarde, contraste avec le reste du décor, celui du sable gris (lemonde.fr, le 9 janvier 2016), une chaussure d'homme certainement perdue par un migrant, laissée dans le sable humide parsemé de lambeaux de coquillages et de petits cailloux (gosc.pl, le 8 juin 2015), un enfant au bord de la mer enroulé dans une couverture qui le protège du froid (lemonde.fr, le 3 mars 2016). On y joue sur le paramètre de quantité, soit on met en scène un ensemble homogène d'objets ou d'individus, soit on place un seul élément, la portée axiologique à laquelle contribuent les symboles étant également très élevée. La relation entre le verbal et le visuel est celle de complémentarité (Tomaszkiewicz, 2006, pp. 60-61), les deux ayant le statut égal au niveau de la construction du sens dans l'énoncé. Pourtant, l'image joue directement sur les émotions des récepteurs et la dimension perlocutoire s'instaure immédiatement à chaque fois quand les images explicitées les mettent dans un état d'angoisse. Toutes les images symboliques analysées créent un temps discontinu et imprévisible puisqu'elles sont dépourvues de marques spatio-temporels précis. Ainsi le récepteur ne peut modifier individuellement les conditions de vérité du message transmis, le contenu vériconditionnel de l'énoncé étant de nature universelle.

\subsubsection{TOPOÏ DU CONFLIT (ANALOGIE À LA GUERRE QUI MENACE L'EUROPE)}

La médiatisation du problème de la migration en Europe s'effectue à l'aide de la métaphore de la guerre. Les lexèmes qui servent à dénommer les événements évoquent le champ d'un conflit entre deux actants : guerre (20-25), lutte (26), bataille (28), opération (27). Certains emplois sont marqués par de fortes valeurs implicites véhiculant des jugements de valeur, comme dans l'exemple 20 , où la guerre sainte 
évoque le topos du conflit mené au nom d'une raison suprême et approuvé par l'autorité divine. Fréquents sont les emplois généralisants où le patient du procès est suggéré comme collectif (Europe) (20-22).

(20) Święta wojna z islamem (wyborcza.pl, le 26 septembre 2015) [fr. Guerre sainte contre l'islam]

(21) Tusk o uchodźcach: Jesteśmy świadkami narodzin nowej formy wojny (wyborcza.pl, le 6 octobre 2015) [fr. Tusk sur les réfugiés : Nous assistons à la naissance d'une nouvelle forme de guerre]

(22) À l'origine de l'immigration clandestine : la guerre (fr.sputniknews.com, le 21 avril 2015)

(23) Immigration. L'Europe part en guerre contre les trafiquants et oublie les quotas de migrants (www.courrierinternational.com, le 19 mai 2015)

(24) L'immigration, une guerre moderne contre l'Europe (www.medias-presse.info, le 1 septembre 2015)

(25) Hongrie : une « atmosphère de guerre » contre les migrants (lemonde.fr, le 15 septembre 2015)

(26) L'Italie durcit sa lutte contre l'immigration (lemonde.fr, le 5 novembre 2010)

(27) Migrants: «À Lesbos, c'est l'opération grand nettoyage » (lemonde.fr, le 29 mars 2016)

(28) À Paris, l'autre bataille de Stalingrad des migrants (lemonde.fr, le 19 mars 2016)

La guerre exige deux opposants en action (modalité d'interaction). L'agentivité du premier se manifeste au moment où il entreprend une marche vers l'Europe, un assaut de l'Europe ou sa prise (29-36). Celle du second, quand il essaie d'y faire face. Il se barricade, il procède à la construction de murs afin de repousser l'ennemi (37-41). L'Europe apparaît en tant que forteresse bâtie afin de résister à l'exode (42-44). L'emploi des verbes à l'aspect inaccompli : pol. szturmować (31, 33, 35, 36), fr. marcher (29), s'emmurer (37), se barricader (38) ainsi que des noms déverbaux : pol. szturm $(32,34)$ implique une représentation dynamique des faits présentés. Les mots désignant les sentiments qui accompagnent l'événement en cours sont fortement liés à la peur, à la panique même (46), surtout quand on est en première ligne d'attaque (45) ou lorsqu'on subit une perte déclarée totale (48).

(29) Des milliers de migrants marchent vers l'Europe (lepoint.fr, le 23 août 2015)

(30) L'assaut des immigrants en Espagne (fr.euronews.com, le 14 août 2014)

(31) Przez Macedonię i Serbię do lepszego życia. Tak imigranci szturmują Europę (dziennik.pl, le 18 juillet 2015) [fr. Par la Macédoine et la Serbie pour une vie meilleure. C'est ainsi que les migrants prennent l'assaut de l'Europe]

(32) Szturm na Europę (newsweek.pl, le 5 mars 2011) [fr. L'assaut de l'Europe]

(33) Unijna agencja: Nielegalni imigranci szturmują Europę (newsweek.pl, le 14 mai 2014) [fr. Agence de l'UE : Les migrants illégaux à l'assaut de l'Europe]

(34) Szturm na tunel pod kanałem La Manche. Tysiące imigrantów (wprost.pl, le 29 juillet 2015) [fr. Des milliers d'immigrants à l'assaut du tunnel sous la Manche] 
(35) Imigranci ryzykują życie szturmując Eurotunel (newsweek.pl, le 31 mai 2015) [fr. Les migrants risquent leur vie en partant à l'assaut de l'Eurotunnel]

(36) Imigranci szturmują zasieki na granicy z Macedonią. Policja użyła gazu łzawiącego [WIDEO] (Newsweek, le 29 février 2016) [fr. Les migrants à l'assaut des barbelés à la frontière de la Macédoine. La police a utilisé des gaz lacrymogènes]

(37) Migrants : quand l'Europe s'emmure (lemonde.fr, le 3 septembre 2015)

(38) Contre les migrants, la Bulgarie se barricade (lemonde.fr, le 24 mars 2016)

(39) Wielki Mur Weggierski (newsweek.pl, le 19 juin 2015) [fr. La Grande Muraille de Hongrie]

(40) Państwa UE chcą stworzyć „,mur prawny”, by powstrzymać napływ migrantów (wyborcza.pl, le 19 février 2016) [fr. Les pays de l'UE veulent créer un « mur légal » pour arrêter l'afflux de migrants]

(41) Węgry zbudują czterometrowy mur na granicy z Serbią. 175-kilometrowy płot ma uniemożliwić nielegalnym migrantom przedostanie się na północ Europy. (newsweek.pl, le 19 juin $2015^{5}$ ) [fr. La Hongrie va construire un mur d'une hauteur de 4 mètres à la frontière avec la Serbie. Les $175 \mathrm{~km}$ de clôture ont pour but d'empêcher les migrants illégaux d'entrer dans le nord de l'Europe]

(42) Europejska forteca, czyli imigranckie piekło na ziemi (newsweek.pl, le 14 octobre 2013) [fr. L'Europe forteresse ou l'enfer sur terre pour les migrants]

(43) To początek exodusu. Wypędzeni przez suszę i wojny dobijają się do twierdzy Europa (wyborcza.pl, le 10 septembre 2015) [fr. C'est le début de l'exode. Chassés par la sécheresse et les guerres, ils s'en vont vers l'Europe forteresse]

(44) L'Europe forteresse tombe le masque (liberation.fr, le 7 mars 2016 ${ }^{6}$ )

(45) Migrants en Europe : l'Italie en première ligne (france24.com, le 10 juin 2015)

(46) L'Europe cède à la panique dans le dossier des migrants (lemonde.fr, le 25 février 2016)

(47) Migrants : pacte sans gloire entre l'Union européenne et la Turquie (lemonde.fr, le 19 mars 2016)

(48) La nuit où Angela Merkel a perdu l'Europe (lemonde.fr, le 16 mars 20167)

Les documents iconiques, les photos, sont toujours véhiculaires du sens car elles ont pour fonction la co-construction du discours ainsi que sa légitimation. L'événement est construit à la base d'un parallélisme (Tomaszkiewicz, 2006, pp. 61-62) qui s'établit entre le verbal et le visuel, deux codes sémiotiques distincts et pourtant coordonnés au sein du discours analysé grâce à la reprise du topos du conflit, et celle-ci non quasiment identique. Le plus souvent on y cadre un groupe de migrants qui passent des obstacles afin d'accéder à l'Europe. On y voit des agents de surveil-

\footnotetext{
${ }^{5}$ Le texte constitue un sous-titre mis dans un article intitulé Wielki Mur Wegierski [fr. La Grande Muraille de Hongrie].

${ }^{6}$ Après les négociations avec la Turquie dont le thème était la réduction de la migration vers l'Europe.

${ }^{7}$ À propos de l'accord avec la Turquie signé le 18 mars 2016 sur la réduction de la migration vers l'Europe.
} 
lance à la frontière (wprost.pl, le 21 octobre 2015), des murs, des grilles qui empêchent l'entrée (wyborcza.pl, le 27 mars 2016; humanité.fr, 02.01.2016), des assauts organisés par les migrants, entre autres, aux murs, aux trains (fr.euro news.com, le 14 août 2014 ; wiadomosci.dziennik.pl, le 18 juillet 2015), des batailles avec la police (wiadomosci.dziennik.pl, 03.12.2015; lexpress.fr, 13.04.2016), des camps ainsi que des faux camps organisés par les migrants, entre autres, sur la voie ferroviaire (lemonde.fr, le 18 mars 2016).

\section{CONCLUSIONS}

Premièrement, le thème évoqué est de grande importance dans la politique européenne, il ouvre un débat social à plusieurs niveaux où de nombreux intérêts s'entremêlent : politique, économique, social, culturel. Deuxièmement, la médiatisation de l'événement s'effectue à l'aide de schémas perceptifs, ce qui a une influence sur sa perception sociale. On y voit le recours à la métaphoricité, l'analogie à l'inondation générée par une force naturelle, et à la guerre, en tant que conflit humain. Le cadre métaphorique mis en scène permet d'impliquer à l'événement une dimension argumentative et déclencher une évaluation lors du débat mené au sein de la société. L'événement subit un jugement de valeur négatif dès qu'il est catégorisé en tant que danger. Qu'on le compare à une inondation ou qu'on le conceptualise en tant que guerre, l'image reçue est dramatique. Certes, l'information sur l'immigration en Europe intègre au cours de la médiatisation un circuit d'idéologisation, celleci étant conforme aux principes de la civilisation occidentale, parmi lesquels la paix et la liberté sont les plus demandés ${ }^{8}$. Selon Bugnot, le caractère négatif, dominant l'image médiatisée sur le problème de la migration vers l'Europe, a sa source dans l'idée d'une menace pour la stabilité sociale, économique et politique, et dans la peur de l'Autre (Bugnot, 2012). « Le caractère négatif dominant est ce qui frappe au premier abord. Les angles (1) l'idée d'une menace pour la stabilité politique, (2) la peur de l'Autre et (3) l'ethnocentrisme et le repli identitaire nous parlent d'un monde conflictuel et polarisé (...) » (ibidem, 2012, para. 26). La situation paraît beaucoup plus compliquée face à un Autre qui n'est plus un individu à part, mais un nombre énorme d'individus, une vague d'êtres humains en fuite. La médiatisation de la crise des migrants, telle qu'on peut le voir au résultat de l'analyse effectuée, nous montre une doxa selon laquelle l'idée de paix et celle de liberté jouent un rôle de principes catégoriels dans l'évaluation de la crise. La construction de l'événement par l'analogie aux faits dramatiques, inscrits comme tels dans le réservoir du savoir collectif, constitue le pilier argumentatif sur lequel repose tout le jugement implicite. Le schéma perceptif ainsi créé marque l'espace public de façon que, bien qu'il soit

\footnotetext{
${ }^{8}$ L'occident est défini en tant que topos imaginaire (Amar, 2010).
} 
réfuté par certains, il sera certainement admis par d'autres. Les images construites exploitent des schémas perceptifs sortis du bagage cognitif commun, ce qui les rend plus accessibles. Ainsi, les médias d'information procurent-ils à toute instance de réception un instrument d'appréhension, d'organisation et de compréhension sociale de l'événement en question. 


\section{BIBLIOGRAPHIE}

Amar Diaz, M. (2010). El velo de Occidente. Revista Hoja de Ruta, 32. Retrieved from http://www. hojaderuta.org/ver_articulos.php?id_texto $=569 \&$ id_revista $=42$

Amossy, R. (2006). L'argumentation dans le discours. Paris : Armand Colin.

Amossy, R., Herschberg Pierrot, A. (1997). Stéréotypes et clichés. Langue, discours, société. Paris : Nathan.

Anscombre, J.-C. (1995). Théorie des topoï. Paris : Kimé.

Augé, M. (1992). Non-lieux, introduction à une anthropologie de la surmodernité. Paris : Le Seuil.

Bugnot, M.-A. (2012). Traduction des discours sur l'islam dans la presse de France et d'Espagne. Meta, 57(4), pp. 977-996. Retrieved from www.erudit.org/revue/meta/2012/v57/n4/1021228ar.html. DOI: $10.7202 / 1021228 \mathrm{ar}$

Calabrese, L. (2009). Nommer un événement ou les marges du sens dans les désignations médiatiques : l'exemple de la canicule. In I. Evrard, M. Pierrard, L. Rosier, D. van Raemdonck (éds), Le sens en marge. Représentations linguistiques et observables discursifs (pp. 15-28). Paris : L'Harmattan.

Calabrese-Steimberg, L. (2011). De Hiroshima aux Twin Towers : les désignants d'événements, une mémoire de l'actualité ? Itinéraires : littérature, textes, cultures, 2. Retrieved from https://itine raires.revues.org/157

Calabrese, L. (2013). L'événement en discours. Presse et mémoire sociale. Louvain-la-Neuve : Academia-L'Harmattan.

Charaudeau, P. (2005). Les médias et l'information. L'impossible transparence du discours. Bruxelles : De Boeck.

Kluszczyński, R. W. (2008). Doświadczenie - pamięć - tożsamość. Doświadczenie medialne jako fundament hybrydycznej tożsamości. In A. Zeidler-Janiszewska, R. Nycz (éds), Nowoczesność jako doświadczenie. Dyscypliny - paradygmaty - dyskursy (pp. 170-184). Warszawa : Wydawnictwo Szkoły Wyższej Psychologii Społecznej „Academica”.

Lugrin, G. (2001). Le mélange des genres dans l'hyperstructure. Semen, 13. Retrieved from http://semen. revues.org/2654

Siblot, P. (2001). De la dénomination à la nomination. Cahiers de praxématique, 36. Retrieved from http://praxematique.revues.org/368

Sini, L. (2015). Événements, discours, médias : réflexions à partir de quelques travaux récents. Argumentation et Analyse du Discours, 14. Retrieved from http://aad.revues.org/1912. DOI: 10.4000/aad.1912

Thomson, J. B. (1995). The Media and Modernity: A Social Theory of the Media. Stanford : University Press.

Tomaszkiewicz, T. (2006). Przekład audiowizualny. Warszawa : Wydawnictwo Naukowe PWN.

Veniard, M. (2007). La nomination d'un événement dans la presse quotidienne nationale. Une étude sémantique et discursive : la guerre en Afghanistan et le conflit des intermittents dans "Le Monde " et « Le Figaro » (thèse de doctorat en sciences du langage). Université Sorbonne Nouvelle, Paris III.

Veniard, M. (2013). La nomination des événements dans la presse. Essai de sémantique discursive. Besançon : Presses universitaires de Franche-Comté. 